\title{
Soft gluon resummation in Drell-Yan dilepton production at small transverse momentum: spin asymmetry and a novel asymptotic formula
}

\author{
Hiroyuki Kawamura* \\ Radiation Laboratory, RIKEN, Wako 351-0198, Japan \\ E-mail: hiroyuki@ribf.riken.jp
}

\section{Jiro Kodaira ${ }^{\dagger}$}

Theory Division, KEK, Tsukuba 305-0801, Japan

\section{Kazuhiro Tanaka}

Department of Physics, Juntendo University, Inba, Chiba 270-1695, Japan

E-mail.tanakakesakura.juntendo.ac.jp

\begin{abstract}
We discuss the double-spin asymmetry $\mathscr{A}_{T T}\left(Q_{T}\right)$ in the transversely polarized Drell-Yan process at small transverse-momentum $Q_{T}$ of the produced dilepton. The soft gluon corrections relevant for small $Q_{T}$ are resummed to all orders in $\alpha_{s}$, up to the next-to-leading logarithmic accuracy. We show that the soft gluon corrections largely cancel in the spin asymmetry, but the significant corrections still remain. The asymmetries $\mathscr{A}_{T T}\left(Q_{T}\right)$ are calculated for $p p$ collision at RHIC and J-PARC, and for $p \bar{p}$ collision at GSI. A novel asymptotic formula for $\mathscr{A}_{T T}\left(Q_{T}\right)$ at small $Q_{T}$ is presented, which provides a new approach to extract the transversity $\delta q(x)$ from the experimental data.
\end{abstract}

8th International Symposium on Radiative Corrections (RADCOR)

October 1-5 2007

Florence, Italy

* Speaker.

${ }^{\dagger}$ Deceased. 


\section{Introduction}

The transversely polarized Drell-Yan process (tDY), $p^{\uparrow}+p^{\uparrow} \rightarrow l+\bar{l}+X, p^{\uparrow}+\bar{p}^{\uparrow} \rightarrow l+\bar{l}+X$, is one of the processes where we can access the chiral-odd transversity distributions $\delta q(x)$. It can be measured at the ongoing experiments at RHIC and the possible future experiments at J-PARC, GSI, etc. In this article, we consider the double-spin asymmetry for tDY, especially at the small transverse momentum $Q_{T}$ of the dilepton, where the bulk of the production occurs. When $Q_{T}$ is much smaller than the invariant mass of the dilepton, $Q$, the soft gluon corrections proportional to $\alpha_{s}^{n} \ln ^{m}\left(Q^{2} / Q_{T}^{2}\right)(m \leq 2 n-1)$ are enhanced and have to be resummed to all orders of perturbation theory to obtain a reliable prediction. In [1, 2], we studied the double transverse-spin asymmetry $\mathscr{A}_{T T}\left(Q_{T}\right)$ at small $Q_{T}$ using the result of [3], where the $Q_{T}$ spectrum of the dilepton for tDY was calculated performing the soft gluon resummation up to the next-to-leading logarithmic (NLL) accuracy. In the following, we will summarize the main results obtained in those works.

\section{The double-spin asymmetry for tDY at a measured $Q_{T}$}

The spin-dependent $\left(\Delta_{T} d \sigma \equiv\left(d \sigma^{\uparrow \uparrow}-d \sigma^{\uparrow \downarrow}\right) / 2\right)$ and spin-independent $\left(d \sigma \equiv\left(d \sigma^{\uparrow \uparrow}+d \sigma^{\uparrow \downarrow}\right) / 2\right)$ parts of the tDY cross section with the NLL soft gluon resummation are expressed as

$$
\frac{\left(\Delta_{T}\right) d \sigma}{d Q^{2} d Q_{T}^{2} d y d \phi}=(\cos (2 \phi) / 2) \frac{2 \alpha^{2}}{3 N_{c} S Q^{2}}\left[\left(\Delta_{T}\right) \tilde{X}^{\mathrm{NLL}}\left(Q_{T}^{2}, Q^{2}, y\right)+\left(\Delta_{T}\right) \tilde{Y}\left(Q_{T}^{2}, Q^{2}, y\right)\right],
$$

where $\sqrt{S}$ and $y$ are the total energy and dilepton's rapidity in the hadron CM system, and the factor $\cos (2 \phi) / 2$ for the spin-dependent part shows the characteristic dependence on the azimuthal angle $\phi$ of one of the outgoing leptons with respect to the incoming nucleon's spin axis. The first term $\left(\Delta_{T} \tilde{X}^{\mathrm{NLL}}\right.$ and $\left.\tilde{X}^{\mathrm{NLL}}\right)$ corresponds to the NLL resummed component which contains the first three towers of the logarithmically-enhanced corrections, $\alpha_{s}^{n} \ln ^{m}\left(Q^{2} / Q_{T}^{2}\right) / Q_{T}^{2}(m=2 n-1,2 n-2$, and $2 n-3)$, and the second term $\left(\Delta_{T} \tilde{Y}\right.$ and $\left.\tilde{Y}\right)$ is the remaining non-enhanced component of $O\left(\alpha_{s}\right)$ and is determined such that the expansion of (2.1) to $O\left(\alpha_{s}\right)$ reproduces the corresponding leadingorder (LO) cross section for $Q_{T}>0$. Accordingly, we call (2.1) the "NLL+LO" cross sections. $\Delta_{T} \tilde{X}^{\mathrm{NLL}}$ and $\tilde{X}^{\mathrm{NLL}}$ are obtained through various elaborations [4, 3, 1] of the Collins-Soper-Sterman resummation formalism [5], and $\Delta_{T} \tilde{X}^{\mathrm{NLL}}$ is given by the integral over the impact parameter $b$, conjugate to $Q_{T}$, as

$$
\begin{array}{rl}
\Delta_{T} \tilde{X}^{\mathrm{NLL}}\left(Q_{T}^{2}, Q^{2}, y\right)=\int_{\mathscr{C}} & d b \frac{b}{2} J_{0}\left(b Q_{T}\right) e^{S(b, Q)-g_{N P} b^{2}}\left[\delta H\left(x_{1}^{0}, x_{2}^{0} ; \frac{b_{0}^{2}}{b^{2}}\right)\right. \\
& \left.+\frac{\alpha_{s}\left(Q^{2}\right)}{2 \pi}\left\{\int_{x_{1}^{0}}^{1} \frac{d z}{z} \Delta_{T} C_{q q}^{(1)}(z) \delta H\left(\frac{x_{1}^{0}}{z}, x_{2}^{0} ; \frac{b_{0}^{2}}{b^{2}}\right)+\left(x_{1}^{0} \leftrightarrow x_{2}^{0}\right)\right\}\right],
\end{array}
$$

where the DY scaling variables are denoted as $x_{1,2}^{0}=\sqrt{Q^{2} / S} e^{ \pm y}, J_{0}\left(b Q_{T}\right)$ is a Bessel function and $b_{0}=2 e^{-\gamma_{E}}$ with $\gamma_{E}$ the Euler constant. $\delta H$ denotes the sum of the products of the NLO transversity distributions: $\delta H\left(x_{1}, x_{2} ; \mu^{2}\right)=\sum_{q} e_{q}^{2}\left[\delta q\left(x_{1}, \mu^{2}\right) \delta \bar{q}\left(x_{2}, \mu^{2}\right)+\left(x_{1} \leftrightarrow x_{2}\right)\right]$ for $p p$ collision and $\delta H\left(x_{1}, x_{2} ; \mu^{2}\right)=\sum_{q} e_{q}^{2}\left[\delta q\left(x_{1}, \mu^{2}\right) \delta q\left(x_{2}, \mu^{2}\right)+\delta \bar{q}\left(x_{1}, \mu^{2}\right) \delta \bar{q}\left(x_{2}, \mu^{2}\right)\right]$ for $p \bar{p}$ collision. Note that there is no gluon transversity distribution. The Sudakov factor $e^{S(b, Q)}$ resums the enhanced logarithmic corrections up to the NLL level and is common for the polarized and unpolarized cross 

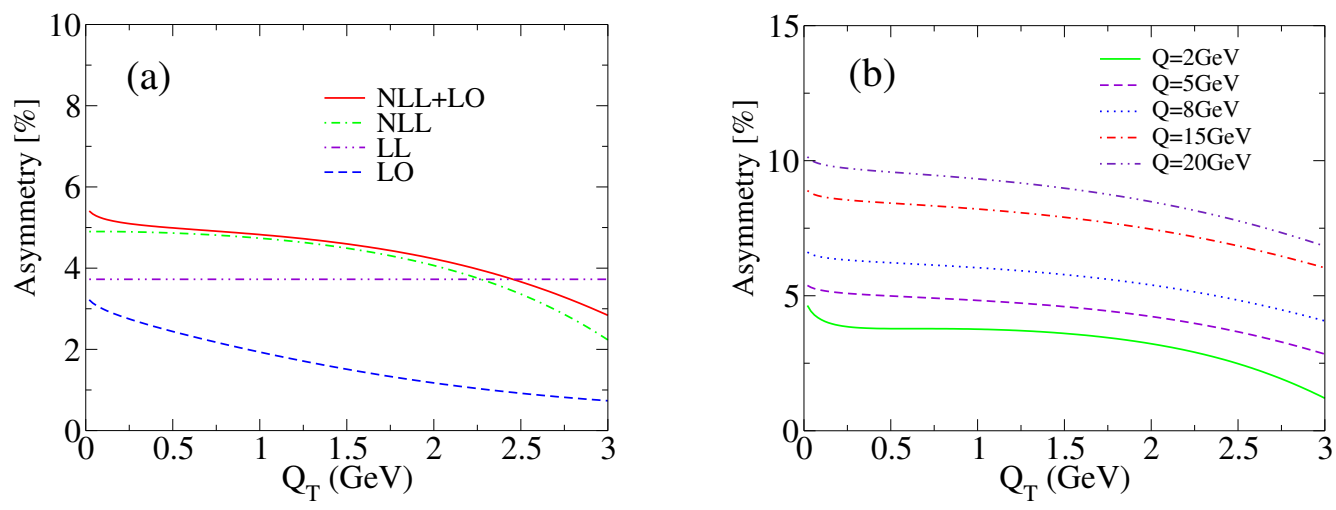

Figure 1: The asymmetries at $\phi=0$ for $p p$ collision with RHIC kinematics, $\sqrt{S}=200 \mathrm{GeV}$ and $y=2$, in (a) various accuracy for $Q=5 \mathrm{GeV}$ and (b) the NLL+LO accuracy for various $Q$.

sections. Using $\lambda=\beta_{0} \alpha_{s}\left(Q^{2}\right) \ln \left(Q^{2} b^{2} / b_{0}^{2}+1\right)$ with $\beta_{0}=\left(11 N_{c}-2 N_{f}\right) /(12 \pi)$, the exponent is obtained as $S(b, Q)=\frac{1}{\alpha_{s}\left(Q^{2}\right)} h^{(0)}(\lambda)+h^{(1)}(\lambda)$, where $h^{(0)}(\lambda)=\left(A_{q}^{(1)} / 2 \pi \beta_{0}^{2}\right)[\lambda+\ln (1-\lambda)]$ with $A_{q}^{(1)}=2 C_{F}=\left(N_{c}^{2}-1\right) / N_{c}$ collects the LL contributions and $h^{(1)}(\lambda)$ collects the NLL contributions. The explicit form of $h^{(1)}(\lambda)$ as well as the coefficient function at the NLL level, $\Delta_{T} C_{q q}^{(1)}(z)$, is found in [3]. The $b$ dependence of $\delta H\left(x_{1}, x_{2} ; b_{0}^{2} / b^{2}\right)$ associated with the NLO evolution of the parton distributions is also organized in terms of $\lambda[4,1]$. The contour $\mathscr{C}$ in (2.2) is taken in the complex $b$ space to avoid the singularity at $\lambda=1$ (see [3, 1]) and we have introduced a Gaussian smearing function $e^{-g_{N P} b^{2}}$ with a parameter $g_{N P}$ to complement the nonperturbative effects from the extremely large $|b|$ region. The resummed component of the unpolarized cross section, $\tilde{X}^{\mathrm{NLL}}$, is obtained similarly. The explicit expressions for $\tilde{X}^{\mathrm{NLL}}, \Delta_{T} \tilde{Y}$ and $\tilde{Y}$ are found in [1, 3].

The NLL+LO asymmetry $\mathscr{A}_{T T}\left(Q_{T}\right)$ is defined by

$$
\mathscr{A}_{T T}\left(Q_{T}\right)=\frac{\Delta_{T} d \sigma / d Q^{2} d Q_{T}^{2} d y d \phi}{d \sigma / d Q^{2} d Q_{T}^{2} d y d \phi}=\frac{1}{2} \cos (2 \phi) \frac{\Delta_{T} \tilde{X}^{\mathrm{NLL}}\left(Q_{T}^{2}, Q^{2}, y\right)+\Delta_{T} \tilde{Y}\left(Q_{T}^{2}, Q^{2}, y\right)}{\tilde{X}^{\mathrm{NLL}}\left(Q_{T}^{2}, Q^{2}, y\right)+\tilde{Y}\left(Q_{T}^{2}, Q^{2}, y\right)}
$$

for measured $Q_{T}, Q, y$ and $\phi$. When the resummed components are expanded to $O\left(\alpha_{s}\right)$, the above asymmetry reduces to the LO prediction $\mathscr{A}_{T T}^{\mathrm{LO}}\left(Q_{T}\right)$ for $Q_{T}>0$. For comparison, we also introduce the NLL asymmetry $\mathscr{A}_{T T}^{\mathrm{NLL}}\left(Q_{T}\right)=[\cos (2 \phi) / 2] \Delta_{T} \tilde{X}^{\mathrm{NLL}} / \tilde{X}^{\mathrm{NLL}}$ and the LL asymmetry $\mathscr{A}_{T T}^{\mathrm{LL}}=$ $[\cos (2 \phi) / 2] \delta H\left(x_{1}^{0}, x_{2}^{0} ; Q^{2}\right) / H\left(x_{1}^{0}, x_{2}^{0} ; Q^{2}\right)$, where the latter is obtained from $\mathscr{A}_{T T}^{\mathrm{NLL}}\left(Q_{T}\right)$ by dropping the NLL terms and, for $p p$ collision, $H\left(x_{1}, x_{2} ; \mu^{2}\right)=\sum_{q} e_{q}^{2}\left[q\left(x_{1}, \mu^{2}\right) \bar{q}\left(x_{2}, \mu^{2}\right)+\left(x_{1} \leftrightarrow x_{2}\right)\right]$. Note that $\mathscr{A}_{T T}^{\mathrm{LL}}$ does not depend on $Q_{T}$ since the soft gluon corrections at the LL level cancel out in the asymmetry [1].

In Fig. 1(a), we compare these asymmetries in various accuracy for $p p$ collision at RHIC with $\sqrt{S}=200 \mathrm{GeV}, Q=5 \mathrm{GeV}, y=2$. Here and below, we use the NLO transversity constructed as in [6] and $g_{N P}=0.5 \mathrm{GeV}^{2}$, and set $\phi=0$. The NLL+LO asymmetry $\mathscr{A}_{T T}\left(Q_{T}\right)$ is flat in the small $Q_{T}$ region and is close to $\mathscr{A}_{T T}^{\mathrm{NLL}}\left(Q_{T}\right)$; i.e., $\mathscr{A}_{T T}\left(Q_{T}\right)$ is dominated by the resummed components, $\Delta_{T} \tilde{X}^{\mathrm{NLL}}$ and $\tilde{X}^{\mathrm{NLL}}$. The flat behavior of $\mathscr{A}_{T T}^{\mathrm{NLL}}\left(Q_{T}\right)$ is due to the universality of the soft gluon corrections in the Sudakov factor $e^{S(b, Q)}$ up to the NLL level. Compared with $\mathscr{A}_{T T}^{\mathrm{LL}}, \mathscr{A}_{T T}^{\mathrm{NLL}}\left(Q_{T}\right)$ is enhanced by the contributions at the NLL level. The LO asymmetry $\mathscr{A}_{T T}^{\mathrm{LO}}\left(Q_{T}\right)$ is much smaller 

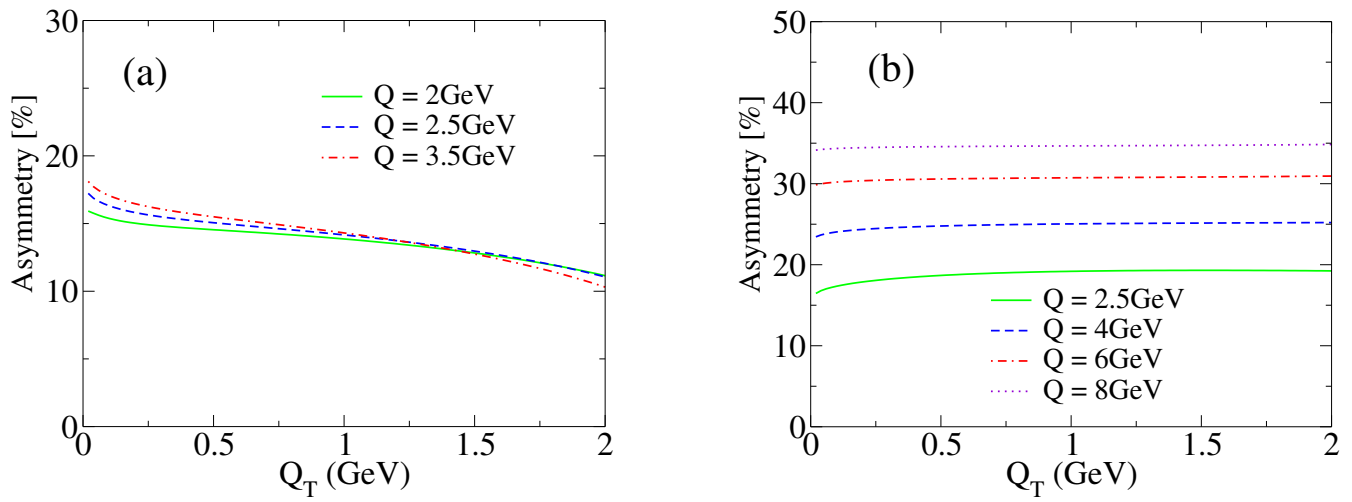

Figure 2: The NLL+LO $\mathscr{A}_{T T}\left(Q_{T}\right)$ of (2.3) at $\phi=0$ for (a) $p p$ collision with J-PARC kinematics, $\sqrt{S}=10$ $\mathrm{GeV}$ and $y=0$, and (b) $p \bar{p}$ collision with GSI kinematics, $\sqrt{S}=14.5 \mathrm{GeV}$ and $y=0$.

than the other asymmetries, indicating that the soft gluon resummation is crucial for the prediction of the asymmetry. Moreover, the NLL+LO asymmetry $\mathscr{A}_{T T}\left(Q_{T}\right)$ is larger than the asymmetry for the $Q_{T}$-integrated cross sections

$$
A_{T T} \equiv \frac{\int d Q_{T}^{2}\left(\Delta_{T} d \sigma / d Q^{2} d Q_{T}^{2} d y d \phi\right)}{\int d Q_{T}^{2}\left(d \sigma / d Q^{2} d Q_{T}^{2} d y d \phi\right)}=\frac{1}{2} \cos (2 \phi) \frac{\delta H\left(x_{1}^{0}, x_{2}^{0} ; Q^{2}\right)+\cdots}{H\left(x_{1}^{0}, x_{2}^{0} ; Q^{2}\right)+\cdots} .
$$

Indeed, $A_{T T}=4.0 \%$ in the present case. In the RHS of (2.4), the ellipses represent the NLO correction terms, and $A_{T T}$ coincides with the NLO asymmetry calculated in [6], because our differential cross sections (2.1) are constructed to satisfy the "unitarity constraint" [4]. This also implies $A_{T T} \simeq \mathscr{A}_{T T}^{\mathrm{LL}}$. The NLL+LO asymmetries $\mathscr{A}_{T T}\left(Q_{T}\right)$ for different values of $Q$ are shown in Fig.1(b). $\mathscr{A}_{T T}\left(Q_{T}\right)$ is flat in all cases and increases as $Q$ increases. This $Q$ dependence is a result of the small- $x$ behavior of the relevant parton distributions, in particular, the steep rise of the unpolarized sea distributions at small $x_{1,2}^{0}=\sqrt{Q / S} e^{ \pm y}$, which enhances the denominator of (2.3) for small $Q$.

Figure 2(a) shows the NLL+LO asymmetries $\mathscr{A}_{T T}\left(Q_{T}\right)$ for $p p$ collision at J-PARC with $\sqrt{S}=$ $10 \mathrm{GeV}, y=0 . \mathscr{A}_{T T}\left(Q_{T}\right)$ are about $15 \%$, irrespective of the value of $Q$, and are larger than those for the RHIC case, since the distributions at the moderate $x$ values are probed at J-PARC [1]. In all the cases in Figs.1 and 2(a) for $p p$ collision, $\mathscr{A}_{T T}\left(Q_{T}\right)$ are larger by $15-30 \%$ than $A_{T T} \simeq \mathscr{A}_{T T}^{\mathrm{LL}}$. Figure 2(b) shows the NLL+LO $\mathscr{A}_{T T}\left(Q_{T}\right)$ for $p \bar{p}$ collision at GSI with $\sqrt{S}=14.5 \mathrm{GeV}, y=0$. The asymmetries are much larger than the previous two cases, since the products of valence distributions are probed at moderate $x$. This fact also leads to $\mathscr{A}_{T T}\left(Q_{T}\right) \simeq A_{T T} \simeq \mathscr{A}_{T T}^{\mathrm{LL}}$ at GSI (see [2] for the detail).

\section{The saddle-point formula for tDY asymmetries at NLL}

For all cases discussed in Figs.1(b) and (2), we actually have $\mathscr{A}_{T T}\left(Q_{T}\right) \simeq \mathscr{A}_{T T}^{\mathrm{NLL}}\left(Q_{T}\right) \simeq$ $\mathscr{A}_{T T}^{\mathrm{NLL}}\left(Q_{T}=0\right)$, similarly as in Fig.1(a). At $Q_{T}=0$, the $b$ integral in (2.2) is controlled by a saddle-point and can be evaluated analytically [1] as

$$
\Delta_{T} \tilde{X}^{\mathrm{NLL}}\left(0, Q^{2}, y\right)=\left[\frac{b_{0}^{2}}{4 Q^{2} \beta_{0} \alpha_{s}\left(Q^{2}\right)} \sqrt{\frac{2 \pi}{\zeta^{(0)^{\prime \prime}}\left(\lambda_{S P}\right)}} e^{-\zeta^{(0)}\left(\lambda_{S P}\right)+h^{(1)}\left(\lambda_{S P}\right)}\right] \delta H\left(x_{1}^{0}, x_{2}^{0} ; \frac{b_{0}^{2}}{b_{S P}^{2}}\right),
$$




\begin{tabular}{|c|c|c|c||c|c|c||c|c|c|}
\hline & \multicolumn{3}{|c||}{ RHIC $(\sqrt{S}=200 \mathrm{GeV}, y=2)$} & \multicolumn{3}{c||}{ J-PARC $(\sqrt{S}=10 \mathrm{GeV}, y=0)$} & \multicolumn{3}{c|}{ GSI $(\sqrt{S}=14.5 \mathrm{GeV}, y=0)$} \\
\hline$Q$ & $5 \mathrm{GeV}$ & $8 \mathrm{GeV}$ & $15 \mathrm{GeV}$ & $2 \mathrm{GeV}$ & $2.5 \mathrm{GeV}$ & $3.5 \mathrm{GeV}$ & $2.5 \mathrm{GeV}$ & $4 \mathrm{GeV}$ & $6 \mathrm{GeV}$ \\
\hline Eq.(3.2) & $5.4 \%$ & $6.6 \%$ & $8.7 \%$ & $14.1 \%$ & $14.5 \%$ & $14.8 \%$ & $20.2 \%$ & $25.6 \%$ & $30.9 \%$ \\
\hline
\end{tabular}

Table 1: $\mathscr{A}_{T T}^{\mathrm{NLL}}\left(Q_{T}=0\right)$ at $\phi=0$ using the saddle-point formula (3.2).

where $\zeta^{(0)}(\lambda) \equiv-\lambda /\left[\beta_{0} \alpha_{S}\left(Q^{2}\right)\right]-h^{(0)}(\lambda) / \alpha_{S}\left(Q^{2}\right)+\left[g_{N P} b_{0}^{2} / Q^{2}\right] e^{\lambda /\left[\beta_{0} \alpha_{s}\left(Q^{2}\right)\right]}$, and the saddle-point value, $\lambda_{S P}=\beta_{0} \alpha_{s}\left(Q^{2}\right) \ln \left(Q^{2} b_{S P}^{2} / b_{0}^{2}+1\right) \simeq \beta_{0} \alpha_{s}\left(Q^{2}\right) \ln \left(Q^{2} b_{S P}^{2} / b_{0}^{2}\right)$, is determined by the condition $\zeta^{(0)^{\prime}}\left(\lambda_{S P}\right)=0$. Note that the saddle-point formula (3.1) is exact up to $O\left(\alpha_{S}\right)$ corrections that actually correspond to the NNLL contributions in the $Q_{T} \approx 0$ region [1]. Similarly, the saddlepoint formula for $\tilde{X}^{\mathrm{NLL}}$ is given by (3.1) with $\delta H\left(x_{1}^{0}, x_{2}^{0} ; b_{0}^{2} / b_{S P}^{2}\right)$ replaced by $H\left(x_{1}^{0}, x_{2}^{0} ; b_{0}^{2} / b_{S P}^{2}\right)$. Therefore, the large radiative corrections included in the square bracket in the RHS of (3.1) cancel out in the asymmetry, and we obtain a remarkably compact formula using the $Q_{T} \rightarrow 0$ limit of the NLL asymmetry:

$$
\mathscr{A}_{T T}\left(Q_{T}\right) \simeq \mathscr{A}_{T T}^{\mathrm{NLL}}\left(Q_{T}=0\right)=\frac{1}{2} \cos (2 \phi) \frac{\delta H\left(x_{1}^{0}, x_{2}^{0} ; b_{0}^{2} / b_{S P}^{2}\right)}{H\left(x_{1}^{0}, x_{2}^{0} ; b_{0}^{2} / b_{S P}^{2}\right)} .
$$

In the RHS, the corrections at the NLL level which survive the cancellation are entirely absorbed into the unconventional scale $b_{0} / b_{S P}$ in the relevant distribution functions, and $b_{0} / b_{S P} \simeq 1 \mathrm{GeV}$ for all cases in Figs. 1 and 2 [1, 2]. This scale $b_{0} / b_{S P}$ in (3.2), instead of $Q$, explains why $\mathscr{A}_{T T}\left(Q_{T}\right)$ is always larger than $\mathscr{A}_{T T}^{\mathrm{LL}}$ or $A_{T T}$ of (2.4) for $p p$ collision [1]. Also, this formula clarifies that the $Q$ dependence of $\mathscr{A}_{T T}\left(Q_{T}\right)$ in Figs.1(b) and 2 reflects the shape of the parton distributions at the scale $b_{0} / b_{S P} \simeq 1 \mathrm{GeV}$. The saddle-point formula (3.2) approaches the exact asymmetry in the $Q \rightarrow \infty$ limit.

In Table 1, we list the values of $\mathscr{A}_{T T}^{\mathrm{NLL}}\left(Q_{T}=0\right)$ obtained from the saddle-point formula (3.2) for the kinematics in Figs.1 and 2. The formula indeed reproduces the NLL+LO $\mathscr{A}_{T T}\left(Q_{T}\right)$ in the flat region to the $10 \%$ accuracy, i.e., to the canonical size of the $\mathscr{O}\left(\alpha_{s}\right)$ corrections associated with the NLL accuracy. It has been demonstrated that certain NNLL corrections to (3.2) can grow for the RHIC kinematics at small $Q$, corresponding to the small- $x$ region, but those corrections are always small for J-PARC and GSI [1, 2]. Therefore, the formula (3.2) is particularly useful for J-PARC and GSI to extract the transversity distributions directly from the data.

The present framework for the soft gluon resummation can be applied to other polarized and unpolarized processes, such as vector boson production at RHIC, LHC, etc.

This work is supported by the Grant-in-Aid for Scientific Research No. B-19340063.

\section{References}

[1] H. Kawamura, J. Kodaira and K. Tanaka, Nucl. Phys. B777 (2007) 203; Prog. Theor. Phys. 118 (2007) 581 [arXive:0709.1752 [hep-ph]].

[2] H. Kawamura, J. Kodaira and K. Tanaka, arXive:0801.0026.

[3] H. Kawamura, J. Kodaira, H. Shimizu and K. Tanaka, Prog. Theor. Phys. 115 (2006) 667. 
[4] G. Bozzi, S. Catani, D. de Florian and M. Grazzini, Phys. Lett. B564 (2003) 65; Nucl. Phys. B737 (2006) 73; ibid. B791 (2007) 1.

[5] J. C. Collins, D. Soper and G. Sterman, Nucl. Phys. B250 (1985) 199.

[6] O. Martin, A. Schäfer, M. Stratmann and W. Vogelsang, Phys. Rev. D57 (1998) 3084; ibid. D60 (1999) 117502. 